\title{
Protective effect of olmesartan against cardiac ischemia/reperfusion injury in spontaneously hypertensive rats
}

\author{
XIN LU ${ }^{1,2}$, YAN-WEN BI ${ }^{1}$, KE-BIAO CHEN ${ }^{2}$ and HONG-YUE WANG ${ }^{2}$ \\ ${ }^{1}$ Department of Cardiovascular Surgery, Qilu Hospital, Shandong University, Jinan, Shandong 250012; \\ ${ }^{2}$ Department of Cardiovascular Surgery, Taian City Central Hospital, Taian, Shandong 271000, P.R. China
}

Received October 4, 2014; Accepted March 5, 2015

DOI: $10.3892 /$ etm.2015.2373

\begin{abstract}
Olmesartan, as a new angiotensin II receptor blocker, has shown beneficial effects on cardiovascular diseases. Nevertheless, the effect of olmesartan on ischemia/reperfusion (I/R) injury in the hypertensive heart has not been investigated. Therefore, the present study aimed to investigate the effect of olmesartan on I/R injury in spontaneously hypertensive rats (SHRs). Experimental groups were designed with a $2 \times 2$ factorial design for olmesartan and I/R effects. In the I/R group, the left anterior descending coronary artery (LAD) was ligated for 40 min followed by a 180 -min reperfusion. In the sham group, SHRs underwent the same surgical procedure as the I/R group, with the exception that the suture passed under the LAD without being tightened. In the Olm-I/R group, the SHRs received olmesartan $(5 \mathrm{mg} / \mathrm{kg})$ for 4 weeks prior to surgery and other procedures were the same as for the I/R group. In the Olm-sham group, the SHRs received olmesartan $(5 \mathrm{mg} / \mathrm{kg})$ for 4 weeks prior to surgery and other procedures were the same as for the sham group. Infarct size was measured for the I/R and Olm-I/R groups. Blood pressure (BP), serum creatine kinase (CK), left ventricular mass index (LVMI), high mobility group box 1 (HMGB1) protein expression levels and hypoxia-inducible factor-1 $\alpha(\mathrm{HIF}-1 \alpha)$ mRNA expression levels were measured for all four groups. Olmesartan significantly reduced BP and LVMI in the olmesartan-treated SHRs compared with those in the SHRs that were not treated with olmesartan. HMGB1 and HIF-1 $\alpha$ expression levels were significantly decreased in the Olm-sham and Olm-I/R groups compared with those in the sham and $\mathrm{I} / \mathrm{R}$ groups, respectively. The proportional increase in HIF-1 $\alpha$ expression due to I/R was greater in the olmesartan-treated rats than in the untreated rats. Serum
\end{abstract}

Correspondence to: Professor Yan-Wen Bi, Department of Cardiovascular Surgery, Qilu Hospital, Shandong University, 107 Wen Hua Xi Road, Jinan, Shandong 250012, P.R. China E-mail: ywbi_qlyy@sina.cn

Key words: spontaneously hypertensive rats, ischemia/reperfusion injury, high mobility group box 1 , hypoxia-inducible factor- $1 \alpha$, olmesartan
CK levels were significantly reduced in the Olm-I/R group compared with those in the I/R group. In conclusion, olmesartan ameliorates left ventricular hypotrophy and protects the heart against I/R injury in addition to lowing BP in SHRs. The protective effect of olmesartan may be partly due to its antioxidative and anti-inflammatory properties.

\section{Introduction}

Ischemic heart disease (IHD) is the leading cause of mortality worldwide (1). The main treatment strategy of IHD is the early reperfusion and restoration of blood flow. However, reperfusion itself contributes to the final myocardial injury via a complex series of events $(2,3)$. Among the various mechanisms that have been reported to explain this phenomenon, inflammation and increased oxidative stress have been reported to be significant contributors to reperfusion injury (4).

High mobility group box 1 (HMGB1) is a ubiquitously expressed nuclear factor and an important mediator of inflammation. Nuclear HMGB1 translocates to the cytoplasm and acts via receptor for advanced glycation end products (RAGE) and/or Toll-like receptors (TLRs) to stimulate various signaling pathways leading to the expression of various leukocyte adhesion molecules and pro-inflammatory cytokines and chemokines (5). A recent study has demonstrated that cardiomyocyte hypertrophy is associated with the increased translocation of HMGB1 from the nucleus to the cytoplasm (6); activation of peroxisome proliferator-activated receptor $\alpha$ (PPAR $\alpha$ ) by fenofibrate inhibits HMGB1 cytoplasmic translocation and prevents the development of cardiac hypertrophy (7). Moreover, HMGB1 acts as an early mediator of inflammation and organ damage in ischemia/reperfusion $(\mathrm{I} / \mathrm{R})$ injury of the heart $(8,9)$.

Hypoxia-inducible factor-1 (HIF-1), a heterodimeric complex containing an oxygen-labile $\alpha$-subunit as well as a constitutively expressed $\beta$-subunit (10), functions as a master regulator of $\mathrm{O}_{2}$ homeostasis by controlling $\mathrm{O}_{2}$ delivery and utilization (11). A recent study demonstrated that HIF-1 $\alpha$ plays a key role in the development of cardiac hypertrophy in response to hypoxic stress (12). In addition, activation of HIF-1 $\alpha$ and its downstream target genes including erythropoietin, heme oxygenase-1, adiponectin and inducible nitric oxide synthase during ischemia is associated with improved myocardial tolerance to acute I/R injury (13). 
Hypertension, as a common cardiovascular risk factor, increases myocardial load/mechanical stress, and may thereby exacerbate the outcome of an I/R insult to the heart (14). Angiotensin II receptor blockers (ARBs) are a class of drugs with increasing use in the treatment of hypertension. Olmesartan, as a new ARB, has shown beneficial effects beyond the lowering of blood pressure (BP). The VIOS study demonstrated that the suppression of the renin-angiotensin system by olmesartan reverses the remodeling and angiosclerosis of small resistance vessels and prevents target organ damage (15). The EUTOPIA trial showed that 6 weeks of olmesartan treatment significantly reduces the levels of inflammatory factors such as hs-CRP, TNF- $\alpha$, MCP-1 and IL-6 (16). The OLIVUS-Ex trial revealed that olmesartan inhibits the progression of atherosclerosis and reduces the risk of major adverse cardiac and cerebrovascular events (17). The study conducted by Swindle et al elucidated that compared with other ARBs (valsartan, losartan and irbesartan), olmesartan is associated with a lower risk of cardiac events and lower healthcare resource utilization and costs (18). However, the results of two randomized trials (ROADMAP and ORIENT) suggest that high-dose olmesartan is associated with increased cardiovascular mortality compared with placebo in diabetic patients (19). The most recent study has demonstrated that even for patients without diabetes, high-dose olmesartan is associated with increased cardiovascular risk (20). Considering that HMGB1 and HIF-1 $\alpha$ are closely associated with left ventricular hypertrophy and I/R injury, it is hypothesized that HMGB1 and HIF- $1 \alpha$ are involved in the effect of olmesartan on left ventricular hypertrophy and I/R injury. Therefore, the present study was conducted to investigate the effect of olmesartan on I/R injury in spontaneously hypertensive rats (SHR) and the changes of HMGB1 and HIF-1 $\alpha$ induced by olmesartan.

\section{Materials and methods}

Ethics statement. All of the rats were handled in accordance with the Guide for the Care and Use of Laboratory Animals published by the US National Institutes of Health (NIH Publication No. 85-23, revised 1996). The experiment was approved by the Animal Care and Use Committee of Taishan Medical University and was carried out in the Key Laboratory of Atherosclerosis of the Universities of Shandong (Taishan Medical University, Taian, China).

Animal housing and surgical preparation. SHRs, aged 12 weeks, were used in this study. The SHRs were provided by Vital River Laboratories (Beijing, China) and were housed in stainless steel cages, six to a cage, with food and water available ad libitum and natural illumination. Half of the SHRs were given conventional feeds for 4 weeks, while others were given olmesartan $(5 \mathrm{mg} / \mathrm{kg})$ by gavage every day for 4 weeks in addition to conventional feeds. BP was assayed every week.

A myocardial infarction model was established as previously described (21). Following an intraperitoneal injection of sodium pentobarbital $(45 \mathrm{mg} / \mathrm{kg})$, the body weight (BW) of each rat was recorded. The rat was fixed in the supine position on an operation platform with a heating pad $\left(36.0-38.0^{\circ} \mathrm{C}\right)$ to maintain body temperature. The animal was tracheally intubated and mechanically ventilated using a small animal ventilator (Animal Respirator ALC-V8; Shanghai Alcott Biotech Co., Ltd., Shanghai, China). The chest was opened by a left thoracotomy through the fourth intercostal space. After removing the pericardium and exposing the heart, a 6-0 monofilament suture was placed around the proximal portion of the left anterior descending coronary artery (LAD) and passed through a short piece of tubing (PE50) to create a reversible snare. Following stabilization of the heart, coronary occlusion was initiated by clamping the snare onto the epicardial surface directly above the coronary artery. Successful occlusion of the LAD was confirmed by the presence of ST segment elevation on an electrocardiogram and a change in ventricular color from fresh-red to dark-red. Following 40 min of occlusion, reperfusion was achieved by loosening the snare and confirmed by a marked hyperemic response at reperfusion.

Experimental protocol. Forty SHRs were randomly divided equally into four groups with a $2 \times 2$ factorial design for olmesartan and I/R effects. In the I/R group, the LAD was ligated for $40 \mathrm{~min}$ followed by a $180 \mathrm{~min}$ reperfusion. In the sham group, the SHRs underwent the same surgical procedures, with the exception that the suture passed under the LAD coronary artery without being tightened. In the Olm-I/R group, the SHRs received olmesartan every day for 4 weeks prior to surgery and other procedures were the same as for the I/R group. In the Olm-sham group, the SHRs received olmesartan every day for 4 weeks prior to surgery and other procedures were the same as for the sham group.

Measurement of tail artery BP. Systolic blood pressure (SBP) and diastolic blood pressure (DBP) were measured in conscious SHRs every week by tail-cuff plethysmography (ALC-NIBP; Shanghai Alcott Biotech Co., Ltd.) prior to surgery. Recordings were started after 5-10 min of acclimation. Three successful measurements were averaged as a single data point.

Measurement of heart rate (HR) and ventricular arrhythmias. Following a stabilization period of $10 \mathrm{~min}$, the HR was taken as the baseline value using a MP150 data acquisition and analysis system (Biopac Systems Inc., Goleta, CA, USA). In addition, the HR was recorded at $15 \mathrm{~min}$ of ischemia and 30 , 60,120 and 180 min of reperfusion.

Ventricular arrhythmias, such as ventricular tachycardia (VT) and fibrillation (VF), were recorded from LAD occlusion to $2 \mathrm{~h}$ of reperfusion. The severity of arrhythmia was quantified by a scoring system as follows: hearts without arrhythmias, 0; premature ventricular beats (PVBs) only, 1; bigeminy/salvos, 2; VT, 3; frequent VT or transient VF, 4; and hearts with frequent or sustained VF or death of the rat, 5 . Scoring of ventricular arrhythmias was conducted in each heart in the I/R and Olm-I/R groups (22).

Measurement of creatine kinase $(C K)$ and left ventricular mass index (LVMI). At the end of the reperfusion period, arterial blood samples were collected through the left carotid artery. Samples were measured using a Creatine Kinase kit (NAC Method; Kehua Bio-engineering Co., Ltd., Shanghai, China) and TBA-120FR automatic biochemistry analyzer 
Table I. Primer sequences for HIF- $1 \alpha$ and GAPDH.

\begin{tabular}{lccc}
\hline Gene & Product $(\mathrm{bp})$ & Forward & Reverse \\
\hline HIF-1 $\alpha$ & 73 & 5'-GACACGGAAACTGAAGACCAA-3' & 5'-CATTAGAAGAGGGGAACATTACATC-3' \\
GAPDH & 140 & 5'-CAAGTTCAACGGCACAGTCAA-3' & 5'-CGCCAGTAGACTCCACGACA-3'
\end{tabular}

HIF-1 1 , hypoxia-inducible factor- $1 \alpha$.

(Toshiba Corporation, Tokyo, Japan) according to the manufacturer's instructions.

Following the collection of the blood samples, the LAD was reoccluded and $1 \mathrm{ml} 2 \%$ Evans blue dye was injected into the right jugular vein to stain the normally perfused region of the heart and delineate the area at risk (AAR). After the body was stained blue, the rat was sacrificed by injection of $10 \%$ potassium chloride through the right carotid artery. The entire heart was excised and rinsed of excess blue dye. The right ventricle and atria were trimmed off. The weight of the remaining left ventricle (LVW) was measured. LVMI was then calculated as the LVW to BW ratio (LVW/BW, mg/g).

Evaluation of $A A R$ and myocardial infarct (MI) size. Evaluation of the MI size was performed in the I/R and Olm-I/R groups as previously described (23). The left ventricle was deep frozen at $-80^{\circ} \mathrm{C}$. The frozen left ventricle was cut into $\sim 6$ sections from apex to base, then all tissues were incubated in a $1 \%$ solution of 2,3,5-triphenyltetrazolium chloride (TTC) for $15 \mathrm{~min}$ at $37^{\circ} \mathrm{C}$. The infarcted tissue was a characteristic white color, whereas the viable tissue was stained red. The slices were compressed between glass plates and scanned into a computer (Epson model G850A; Seiko Epson Corporation, Nagano, Japan). Following magnification, planimetry was carried out using image analysis software (Image-Pro Plus 6.0; Media Cybernetics, Rockville, MD, USA). The risk region and infarct region were calculated by surface area analysis. Following the summation of individual slices, the AAR was expressed as a percentage of the left ventricle and MI size as a percentage of AAR.

$H I F-1 \alpha$ analysis by reverse transcription-quantitative polymerase chain reaction $(R T-q P C R)$. Total microRNAs were extracted from the AAR of myocardial tissue (100 mg) using UNIQ-10 Column Centrifugation TRIzol Total RNA Isolation kit (Sangon Biotech, Shanghai, China) and reverse transcribed into cDNA using Avian Myeloblastosis Virus First Strand cDNA Synthesis kit (Sangon Biotech) according to the manufacturer's instructions.

Analysis of gene expression was studied using RT-qPCR with SYBR Green PCR Master mix (Applied Biosystems Inc., Carlsbad, CA, USA) and the LightCycler 480 Real-Time PCR System (Roche Diagnostics Ltd., Rotkreuz, Switzerland). The reaction program was $95^{\circ} \mathrm{C}$ for $3 \mathrm{~min}$, followed by 40 cycles of $95^{\circ} \mathrm{C}$ for $15 \mathrm{sec}$ and $60^{\circ} \mathrm{C}$ for $40 \mathrm{sec}$. The primer sequences are presented in Table I. The reference gene for HIF-1 $\alpha$ was GAPDH. Values are expressed as relative expression of HIF-1 $\alpha$ normalized to the reference gene, GAPDH. The relative quantification value for microRNA expression was calculated as $2^{-\Delta \Delta \mathrm{Ct}}$ by the comparative $\mathrm{Ct}$ method (24), where $\Delta \Delta \mathrm{Ct}=\left(\mathrm{Ct}_{\mathrm{HIF}-1 \alpha}\right.$ of treated sample-Ct GAPDH of treated sample)-( $\mathrm{Ct}_{\mathrm{HIF}-1 \alpha}$ of control sample- $\mathrm{Ct}_{\mathrm{GAPDH}}$ of control sample).

Western blot analysis. Total proteins were extracted from the AAR of myocardial tissue (200 mg) using Tissue or Cell Total Protein Extraction kit (Sangon Biotech) according to the manufacturer's instructions. Equal amounts of total cellular proteins were resolved by SDS-PAGE and transferred to polyvinylidene fluoride membranes. The membranes were probed with anti-HMGB1 rabbit monoclonal antibody (EPR3507; 1:10,000; Abcam, Cambridge, UK) followed by HRP-conjugated AffiniPure goat anti-rabbit $\mathrm{IgG}(\mathrm{H}+\mathrm{L})$ secondary antibody (1:8,000; Sangon Biotech). The immunoreactive bands were visualized using an Ultrasensitive ECL Chemiluminescence kit (Sangon Biotech). The same membranes were then stripped and reprobed with anti- $\beta$-actin rabbit polyclonal antibody as an internal control (1:800; Sangon Biotech) followed by HRP-conjugated AffiniPure goat anti-rabbit IgG $(\mathrm{H}+\mathrm{L})$ secondary antibody (1:8,000; Sangon Biotech, Shanghai, China). The immunoreactive bands were visualized using a Ultrasensitive ECL Chemiluminescence kit. Optical densities of the bands were scanned and quantified with Quantity One 1-D Analysis Software (Bio-Rad Laboratories Inc., Hercules, CA, USA). HMGB1 expression levels were normalized to the expression of $\beta$-actin.

Statistical analysis. Statistical analysis was performed with SPSS statistical software, version 13.0 (SPSS Inc., Chicago, IL, USA). Data are presented as mean \pm standard deviation (SD). Two-way analysis of variance (ANOVA) was used to analyze the data with a $2 \times 2$ factorial design for olmesartan and I/R effects (on CK, HMGB and HIF-1 $\alpha$ ); independent-samples t-tests were used to test MI size and scores of ventricular arrhythmia in the I/R and Olm-I/R groups and to compare DBP, SBP and LVMI between the olmesartan-treated SHRs and the SHRs that were not treated with olmesartan; repeated-measures ANOVA was applied for comparisons of $\mathrm{HR}$. $\mathrm{P}<0.05$ was considered to indicate a statistically significant difference.

\section{Results}

BP, LVMI, HR and ventricular arrhythmia. Olmesartan significantly decreased SBP,DBP and LVMI in the olmesartan-treated SHRs $(134.75 \pm 10.69,97.06 \pm 10.82$ and $2.22 \pm 0.12 \mathrm{mmHg}$, respectively) compared with the corresponding values in the SHRs that were not treated with olmesartan $(190.86 \pm 14.75$, $134.24 \pm 16.77$ and $2.49 \pm 0.10 \mathrm{mmHg}$, respectively). 
Table II. Heart rate changes with the ischemia/reperfusion process (beats/min).

Ischemia/reperfusion ( $\mathrm{min})$

\begin{tabular}{lllllll}
\cline { 3 - 6 } Groups & Baseline & \multicolumn{1}{c}{15} & \multicolumn{1}{c}{30} & 60 & \multicolumn{1}{c}{120} & 180 \\
\hline Sham & $376 \pm 12$ & $375 \pm 12$ & $369 \pm 13$ & $363 \pm 13$ & $364 \pm 13$ & $357 \pm 15$ \\
I/R & $375 \pm 10$ & $382 \pm 10$ & $371 \pm 10$ & $360 \pm 7$ & $352 \pm 8$ & $342 \pm 7$ \\
Olm-sham & $370 \pm 10$ & $371 \pm 11$ & $369 \pm 9$ & $361 \pm 11$ & $358 \pm 16$ & $356 \pm 15$ \\
Olm-I/R & $371 \pm 6$ & $380 \pm 8$ & $370 \pm 8$ & $357 \pm 6$ & $351 \pm 8$ & $346 \pm 8$ \\
\hline
\end{tabular}

Data are presented as the mean \pm standard deviation. I/R, ischemia/reperfusion; Olm, olmesartan

Table III. Scores of ventricular arrhythmia measured during the ischemia/reperfusion process.

Scores of ventricular arrhythmia [median (interquartile range)]

\begin{tabular}{llc}
\cline { 2 - 3 } Groups & Ischemia & Reperfusion \\
\hline I/R & $1.7(1)$ & $1.2(1.25)$ \\
Olm-I/R & $1.6(1.75)$ & $1(2)$ \\
\hline
\end{tabular}

$\mathrm{I} / \mathrm{R}$, ischemia/reperfusion; Olm, olmesartan.

HR changes with the I/R process in the four groups are shown in Table II. During the reperfusion, there were no significant differences in HR among the four groups $(\mathrm{P}=0.899$, repeated-measures ANOVA), and HR decreased gradually with reperfusion.

Ventricular arrhythmias were observed in the I/R and Olm-I/R groups. There were no significant differences between the I/R and Olm-I/R group in the scores of ventricular arrhythmia during the I/R process (Table III).

$A A R$ and MI size. No significant differences in the AAR, expressed as a percentage of the left ventricular region, were noted between the I/R group and the Olm-I/R group (Fig. 1). The MI size, expressed as a percentage of the AAR, was significantly reduced in the Olm-I/R group $(21.4 \pm 5.9 \%)$ compared with that in the I/R group $(39.3 \pm 3.6 \%)$.

Serum CK level. I/R injury caused a significant increase of the serum CK concentration (Fig. 2). Olmesartan caused a marked reduction of the serum $\mathrm{CK}$ concentration in the Olm-I/R group $(2,399.9 \pm 807.6 \mathrm{U} / \mathrm{l})$ compared with that in the I/R group $(4,365.8 \pm 605.8 \mathrm{U} / \mathrm{l})$ and a substantial but not statistically significant reduction of the serum $\mathrm{CK}$ concentration in the Olm-sham group $(484.3 \pm 177.2 \mathrm{U} / \mathrm{l})$ compared with that in the sham group $(517.5 \pm 135.3 \mathrm{U} / \mathrm{l})$. Interaction between olmesartan and $\mathrm{I} / \mathrm{R}$ was significant $(\mathrm{P}<0.01$, two-way ANOVA).

$H M G B 1$ protein expression. HMGB1 expression levels were elevated in the two I/R groups compared with those in the two sham groups (Fig. 3). Olmesartan significantly decreased the HMGB1 expression levels in the olmesartan-treated SHRs

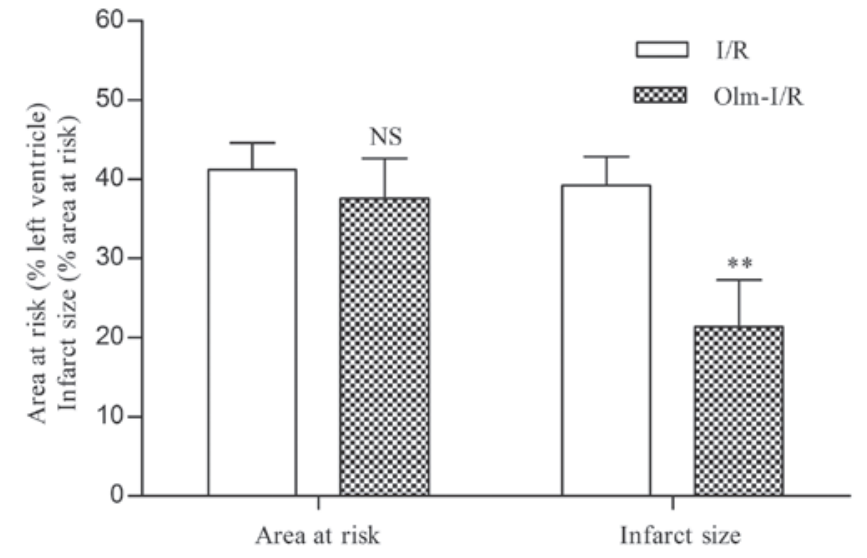

Figure 1. Olmesartan reduced myocardial infarct size in SHRs. The areas at risk were similar for the two groups (NS, not significantly different vs. the I/R group). Infarct size was significantly reduced in the Olm-I/R group (**P $<0.05$ vs. the I/R group). Olm, olmesartan; SHRs, spontaneously hypertensive rats; $\mathrm{I} / \mathrm{R}$, ischemia/reperfusion.

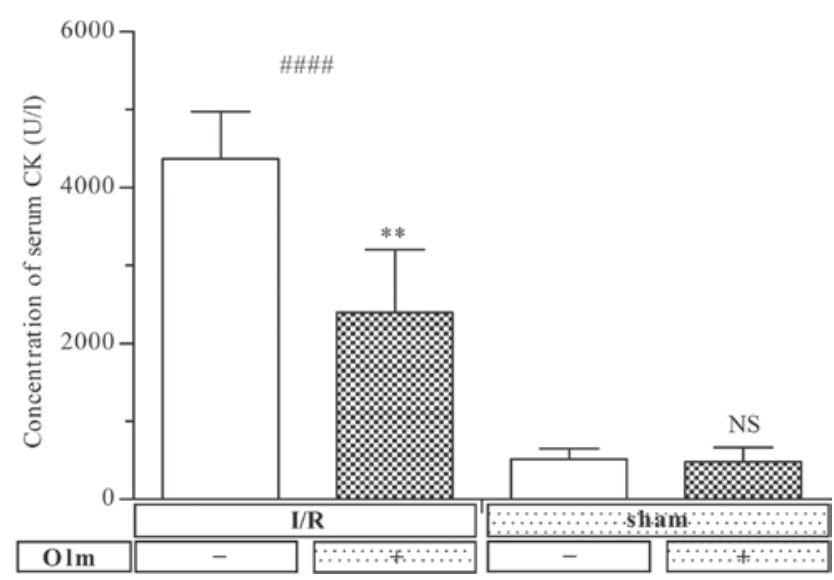

Figure 2. Olmesartan reduced the concentration of serum CK in SHRs with $\mathrm{I} / \mathrm{R}$ injury. I/R injury caused the significant increase in the concentration of serum $C K$ in the $I / R$ groups compared to the sham groups $\left({ }^{\# \# \#} \mathrm{P}<0.001\right)$. The serum concentration of $\mathrm{CK}$ was significantly lower in the Olm-I/R group than in the $\mathrm{I} / \mathrm{R}$ group $(* * \mathrm{P}<0.001)$. The difference in serum $\mathrm{CK}$ concentration was not significant (NS) between the two sham groups $(\mathrm{P}=0.929)$. Olm, olmesartan; CK, creatine kinase; SHRs, spontaneously hypertensive rats; $\mathrm{I} / \mathrm{R}$, ischemia/reperfusion.

compared with those in the SHRs that were not treated with olmesartan (Olm-sham group $0.711 \pm 0.019$ vs. sham group $0.998 \pm 0.009$; and Olm-I/R group $1.251 \pm 0.002$ vs. I/R group 

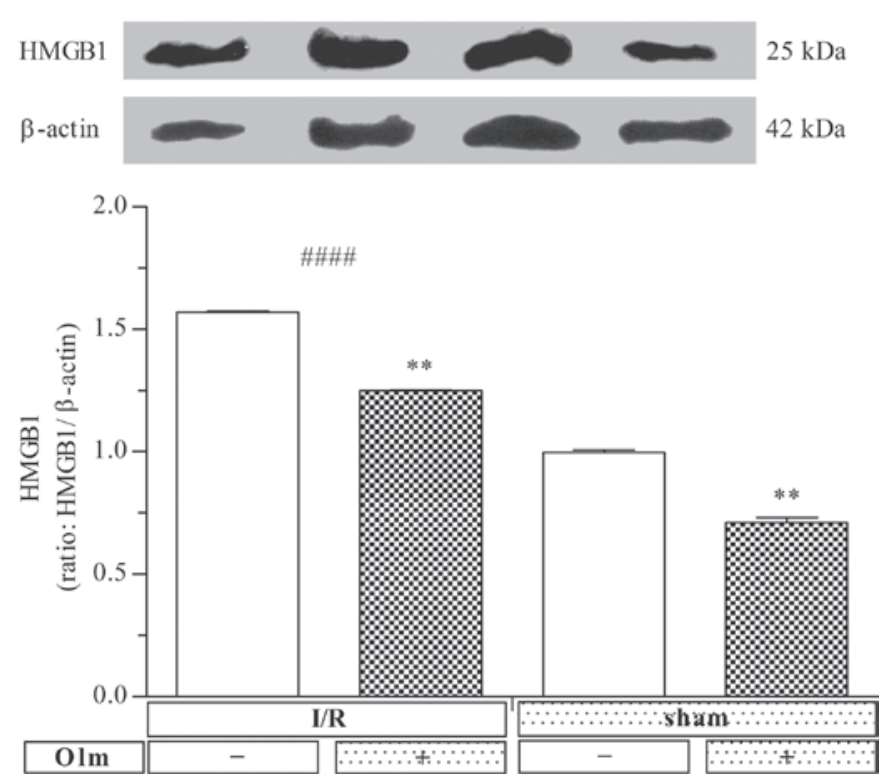

Figure 3. Olmesartan decreased HMGB1 expression in SHRs. Upper panel: Western blot assessment of HMGB1 expression in the area at risk of myocardial tissue. Lower panel: I/R injury increased the expression of HMGB1

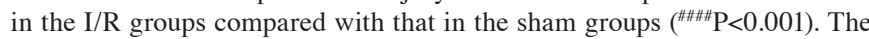
expression of HMGB1 was significantly reduced in the Olm-I/R group compared with that in the $\mathrm{I} / \mathrm{R}$ group $\left({ }^{* *} \mathrm{P}<0.001\right)$ and in the $\mathrm{Olm}$-sham group compared with that in the sham group $\left({ }^{* *} \mathrm{P}<0.001\right)$. Olm, olmesartan; HMGB1, high mobility group box 1; SHRs, spontaneously hypertensive rats; I/R, ischemia/reperfusion.

$1.570 \pm 0.004)$. Interaction between olmesartan and $\mathrm{I} / \mathrm{R}$ was significant $(\mathrm{P}<0.05$, two-way ANOVA).

HIF-1 $\alpha$ mRNA expression levels. The HIF-1 $\alpha$ mRNA expression level was significantly upregulated in the two I/R groups compared with those in the two sham groups (Fig. 4). However, olmesartan downregulated the HIF-1 $\alpha$ mRNA expression levels in the olmesartan-treated SHRs compared with those in the rats that were not treated with olmesartan. Interaction between olmesartan and I/R was significant $(\mathrm{P}<0.05$, two-way ANOVA). Furthermore, it should be noted that the proportional increase of HIF-1 $\alpha$ expression caused by $\mathrm{I} / \mathrm{R}$ injury in the olmesartan-treated rats (Olm-I/R group vs. Olm-sham group, 48.1/21.2) was greater than that in the rats that were not treated with olmesartan (I/R group vs. sham group, 100/58.8).

\section{Discussion}

The renin-angiotensin-aldosterone system (RAAS) plays a major role in the pathophysiology of hypertension and $\mathrm{I} / \mathrm{R}$ injury $(25,26)$. Angiotensin II (Ang II), the key neurohormone of the RAAS, acts through high-affinity cell surface angiotensin II type 1 (AT1) receptors and modulates cardiovascular homeostasis. AT1 receptor activation by Ang II is not only involved in the regulation of BP, water and sodium homeostasis, and the control of other neurohumoral systems, but also leads to excessive production of reactive oxygen species (ROS) and to hypertrophy, proliferation, migration and apoptosis of vascular cells (27). Olmesartan is a nonpeptide Ang II receptor antagonist that selectively and competitively inhibits

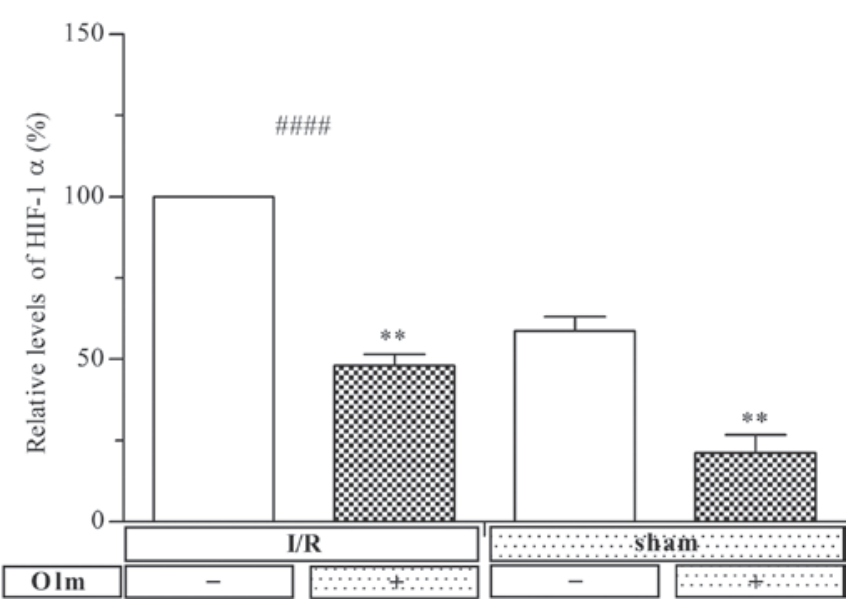

Figure 4. Olmesartan downregulated the expression of HIF-1 $\alpha$ in SHRs. The expression of HIF-1 $\alpha$ was significantly upregulated by I/R injury in the I/R groups vs. the sham groups $\left({ }^{\# \# \# \#} \mathrm{P}<0.001\right)$. Olmesartan downregulated the expression of HIF-1 $\alpha$ in the Olm-I/R group vs. the I/R group $\left({ }^{* *} \mathrm{P}<0.001\right)$ and in the Olm-sham group vs. the sham group $\left({ }^{* *} \mathrm{P}<0.001\right)$. Olm, olmesartan; HIF-1 $\alpha$, hypoxia-inducible factor- $1 \alpha$; SHRs, spontaneously hypertensive rats; $\mathrm{I} / \mathrm{R}$, ischemia/reperfusion.

the AT1 receptor without affecting other receptors regulating the cardiovascular system (28). The present study found that by inhibiting the AT1 receptor and reducing oxidative stress, olmesartan ameliorated left ventricular hypotrophy and protected the heart against I/R injury in addition to lowering BP in SHRs.

Inflammation plays an important role in the pathophysiology of essential hypertension (29). Cardiac hypertrophy and BP development are associated with inflammation (30). In SHRs, increased oxidative stress characterized by increased ROS-generating capacity (31) contributes to HMGB1 release (32) and thereby leads to cardiac hypertrophy through the activation of calcineurin (33). Moreover, overexpression of HIF1- $\alpha$ activated by oxidative stress exists in the hypertrophied heart and may contribute to the pathology of end-stage failure $(34,35)$.

In the present study, 4 weeks of olmesartan treatment significantly reduced left ventricular hypertrophy. The expression levels of HMGB1 and HIF-1 $\alpha$ were reduced significantly in the Olm-sham group compared with those in the sham group. This result indicated that olmesartan reduced oxidative stress by inhibiting the AT1 receptor and thereby reducing the expression of HMGB1 and the expression of HIF-1 $\alpha$ and attenuating LVMI. To the best of our knowledge, the present study demonstrates for the first time that olmesartan, like other ARBs (36), can downregulate the expression level of HMGB1. The study by Fu et al demonstrated that olmesartan attenuated cardiac hypertrophy in SHRs by the calcineurin pathway (37), while the present study demonstrated that the beneficial effect of olmesartan in the reduction of cardiac hypertrophy was due to the suppression of HMGB1 expression and downregulation of HIF-1 $\alpha$ expression.

$\mathrm{I} / \mathrm{R}$ injury is a pathophysiologic process whereby hypoxic tissue damage is accentuated following restoration of the blood supply. Infarct size reduction is the ultimate endpoint of myocardial I/R treatment. Four weeks of olmesartan treatment significantly limited infarct size in the Olm-I/R group 
compared with that in the I/R group. In addition, serum $\mathrm{CK}$ concentration, a surrogate measure of myocardial morphological injury, was also lower in the Olm-I/R group than in the I/R group. However, 4 weeks of olmesartan treatment did not cause a significant reduction in susceptibility to I/R-induced ventricular tachycardia and ventricular fibrillation. In summary, olmesartan exerts cardioprotective effects against I/R injury by limiting infarct size and reducing the serum $\mathrm{CK}$ concentration but does not significantly improve ventricular arrhythmias.

$\mathrm{I} / \mathrm{R}$ injury is characterized by marked oxidative stress and intracellular calcium overload (38), which activates the release of HMGB1 $(32,39)$. HMGB1 then acts via its receptor (RAGE/TL4) to stimulate the release of proinflammatory cytokines, induce NFKB and enhance myocardial injury $(8,9)$. Therefore, HMGB1 can be considered as a marker of $\mathrm{I} / \mathrm{R}$ injury (40). In the present study, I/R injury caused a significant increase of HMGB1 expression. Four weeks of olmesartan treatment, by inhibiting the AT1 receptor and inhibiting oxidative stress, significantly attenuated the expression level of HMGB1 in the Olm-I/R group compared with that in the I/R group. Therefore, the protective effect of olmesartan is partly due to the downregulation of HMGB1 expression. HIF-1 $\alpha$ is expressed in human hearts with acute ischemia or infarction due to increased oxidative stress. HIF-1 $\alpha$ increases the transcription of glucose transporters and glycolytic enzymes to improve glucose utilization, and it also inhibits mitochondrial oxidative metabolism, thereby reducing the generation of ROS under conditions of hypoxia or hypoxia-reoxygenation (11). In the present study, the expression level of HIF-1 $\alpha$ was elevated by I/R injury. While olmesartan suppressed the expression of HIF-1 $\alpha$, the proportional increase in HIF-1 $\alpha$ expression caused by $\mathrm{I} / \mathrm{R}$ injury in the olmesartan-treated rats (Olm-I/R group vs. Olm-sham group) was greater than the proportional increase in HIF-1 $\alpha$ expression caused by I/R injury in the untreated rats (I/R group vs. sham group). Ameliorated left ventricular hypertrophy and reduced oxygen demand in the SHRs of the Olm-I/R group may explain why the increase in HIF-1 $\alpha$ expression due to $\mathrm{I} / \mathrm{R}$ was proportionally greater in the olmesartan-treated rats. Dai et al (41) reported that olmesartan $(3 \mathrm{mg} / \mathrm{kg}$ body weight diluted in $1 \mathrm{ml}$ vehicle) given to Sprague-Dawley rats by a bolus injection via the catheterized left jugular vein at 5 min prior to reperfusion significantly reduced MI size and improved left ventricular contractility; the potential mechanism may be associated with the vasodilating effect observed with the higher dose of olmesartan with subsequent reduction in oxygen demand. The present study demonstrated that olmesartan benefited I/R myocardium in SHRs by two mechanisms: i) by inhibiting HMGB1 and reducing the inflammatory response; ii) by ameliorating left ventricular hypotrophy.

In summary, the present study suggests that 4 weeks of olmesartan treatment ameliorates left ventricular hypotrophy and protects the heart against I/R injury in addition to lowering BP in SHRs. The cardioprotective effect of olmesartan is due to its antioxidative and anti-inflammatory properties. By inhibiting the AT1 receptor and reducing oxidative stress, olmesartan reduces the expression of HMGB1 and downregulates the expression of $\mathrm{HIF}-1 \alpha$, thereby attenuating left ventricular hypertrophy and limiting infarct size. The results indicate that olmesartan may provide a long-term benefit for hypertensive patients with coronary artery disease.

\section{References}

1. Moran AE, Forouzanfar MH, Roth GA, Mensah GA, Ezzati M, Murray CJ and Naghavi M: Temporal trends in ischemic heart disease mortality in 21 world regions, 1980 to 2010: The Global Burden of Disease 2010 study. Circulation 129: 1483-1492, 2014.

2. Piper HM, Meuter K and Schäfer C: Cellular mechanisms of ischemia-reperfusion injury. Ann Thorac Surg 75 (Suppl): S644-S648, 2003

3. Penna C, Mancardi D, Raimonodo S, Geuna S and Pagliaro P: The paradigm of postconditioning to protect the heart. J Cell Mol Med 12: 435-458, 2008.

4. Sharma V, Bell RM and Yellon DM: Targeting reperfusion injury in acute myocardial infarction: A review of reperfusion injury pharmacotherapy. Expert Opin Pharmacother 13: $1153-1175,2012$

5. Nogueira-Machado JA and de Oliveira Volpe CM: HMGB-1 as a target for inflammation controlling. Recent Pat Endocr Metab Immune Drug Discov 6: 201-209, 2012.

6. Funayama A, Shishido T, Netsu S, et al: Cardiac nuclear high mobility group box 1 prevents the development of cardiac hypertrophy and heart failure. Cardiovasc Res 99: 657-664, 2013.

7. Jia Z, Xue R, Liu G, et al: HMGB1 is involved in the protective effect of the PPAR a agonist fenofibrate against cardiac hypertrophy. PPAR Res 2014: 541394, 2014.

8. Andrassy M, Volz HC, Igwe JC, et al: High-mobility group box-1 in ischemia-reperfusion injury of the heart. Circulation 117: 3216-3226, 2008.

9. Zhu H, Li J, Wang S, Liu K, Wang L and Huang L: Hmgb1-TLR4-IL-23-IL-17A axis promote ischemia-reperfusion injury in a cardiac transplantation model. Transplantation 95: 1448-1454, 2013.

10. Wang GL, Jiang BH, Rue EA and Semenza GL: Hypoxia-inducible factor 1 is a basic-helix-loop-helix-PAS heterodimer regulated by cellular $\mathrm{O}_{2}$ tension. Proc Natl Acad Sci USA 92: 5510-5514, 1995.

11. Semenza GL: Hypoxia-inducible factor 1 and cardiovascular disease. Annu Rev Physiol 76: 39-56, 2014.

12. Chu W, Wan L, Zhao D, et al: Mild hypoxia-induced cardiomyocyte hypertrophy via up-regulation of HIF-1 $\alpha$-mediated TRPC signaling. J Cell Mol Med 16: 2022-2034, 2012.

13. Scaffidi P, Misteli T and Bianchi ME. Release of chromatin protein HMGB1 by necrotic cells triggers inflammation. Nature 418: 191-195, 2002.

14. Mozaffari MS, Liu JY, Abebe W and Baban B: Mechanisms of load dependency of myocardial ischemia reperfusion injury. Am J Cardiovasc Dis 3: 180-196, 2013.

15. Smith RD, Yokoyama H, Averill DB, Cooke L, Brosnihan KB, Schiffrin EL and Ferrario CM: The protective effects of angiotensin II blockade with olmesartan medoxomil on resistance vessel remodeling (The VIOS study): Rationale and baseline characteristics. Am J Cardiovasc Drugs 6: 335-342, 2006.

16. Lorenzen JM, Neunhöffer H, David S, Kielstein JT, Haller H and Fliser D: Angiotensin II receptor blocker and statins lower elevated levels of osteopontin in essential hypertension - results from the EUTOPIA trial. Atherosclerosis 209: 184-188, 2010.

17. Hirohata A, Yamamoto K, Miyoshi T, et al: Four-year clinical outcomes of the OLIVUS-Ex (impact of Olmesartan on progression of coronary atherosclerosis: Evaluation by intravascular ultrasound) extension trial. Atherosclerosis 220: 134-138, 2012.

18. Swindle JP, Buzinec P, Iorga SR, Ramaswamy K and Panjabi S: Long-term clinical and economic outcomes associated with angiotensin II receptor blocker use in hypertensive patients. Curr Med Res Opin 27: 1719-1731, 2011.

19. Hamamoto Y and Koshiyama H: ROADMAP and ORIENTAL Trails: The Re-emergence of J-Curve Ghost? Jpn Clin Med 2: 25-28, 2011.

20. Zhou EH, Gelperin K, Levenson MS, Rose M, Hsueh YH and Graham DJ: Risk of acute myocardial infarction, stroke, or death in patients initiating olmesartan or other angiotensin receptor blockers - a cohort study using the Clinical Practice Research Datalink. Pharmacoepidemiol Drug Saf 23: 340-347, 2014.

21. Lim SY, Davidson SM, Hausenloy DJ and Yellon DM: Preconditioning and postconditioning: The essential role of the mitochondrial permeability transition pore. Cardiovasc Res 75: 530-535, 2007.

22. Pourkhalili K, Hajizadeh S, Tiraihi T, Akbari Z, Esmailidehaj M, Bigdeli MR and Khoshbaten A: Ischemia and reperfusion-induced arrhythmias: Role of hyperoxic preconditioning. J Cardiovasc Med (Hagerstown) 10: 635-642, 2009. 
23. Zhao H, Wang Y, Wu Y, et al: Hyperlipidemia does not prevent the cardioprotection by postconditioning against myocardial ischemia/reperfusion injury and the involvement of hypoxia inducible factor-1alpha upregulation. Acta Biochim Biophys Sin (Shanghai) 41: 745-753, 2009.

24. Yoshida A, Suzuki N, Nakano Y, Oho T, Kawada M and Koga T: Development of a 5 'fluorogenic nuclease-based real-time PCR assay forquantitative detection of Actinobacillus actinomycetemcomitans and Porphyromonas gingivalis. J Clin Microbiol 41: 863-866, 2003.

25. Paulis L and Unger T: Novel therapeutic targets for hypertension. Nat Rev Cardiol 7: 431-441, 2010.

26. Dai W and Kloner RA: Potential role of renin-angiotensin system blockade for preventing myocardial ischemia/reperfusion injury and remodeling after myocardial infarction. Postgrad Med 123: 49-55, 2011.

27. Wassmann S and Nickenig G: Pathophysiological regulation of the AT1-receptor and implications for vascular disease. J Hypertens Suppl 24: S15-S21, 2006.

28. Chilman-Blair K and Rabasseda X: Olmesartan, an AT1-selective antihypertensive agent. Drugs Today (Barc) 39: 745-761, 2003.

29. Montecucco F, Pende A, Quercioli A and Mach F: Inflammation in the pathophysiology of essential hypertension. J Nephrol 24 23-34, 2011.

30. Rodríguez-Iturbe B, Ferrebuz A, Vanegas V, Quiroz Y, Mezzano S and Vaziri ND: Early and sustained inhibition of nuclear factor-kappaB prevents hypertension in spontaneously hypertensive rats. J Pharmacol Exp Ther 315: 51-57, 2005.

31. Portaluppi F, Boari B and Manfredini R: Oxidative stress in essential hypertension. Curr Pharm Des 10: 1695-1698, 2004.

32. Tang D, Kang R, Xiao W, et al: Nuclear heat shock protein 72 as a negative regulator of oxidative stress (hydrogen peroxide)-induced HMGB1 cytoplasmic translocation and release. J Immunol 178: 7376-7384, 2007.
33. Su FF, Shi MQ, Guo WG, Liu XT, Wang HT, Lu ZF and Zheng QS: High-mobility group box 1 induces calcineurin-mediated cell hypertrophy in neonatal rat ventricular myocytes. Mediators Inflamm 2012: 805149, 2012

34. Krishnan J, Suter M, Windak R, et al: Activation of a HIF1 alpha-PPARgamma axis underlies the integration of glycolytic and lipid anabolic pathways in pathologic cardiac hypertrophy. Cell Metab 9: 512-524, 2009

35. Shyu KG, Liou JY, Wang BW, Fang WJ and Chang $\mathrm{H}$ Carvedilol prevents cardiac hypertrophy and overexpression of hypoxia-inducible facor-1alpha and vascular endothelial growth factor in pressure-overloaded rat heart. J Biomed Sci 12: 409-420, 2005

36. Kikuchi K, Tancharoen S, Ito T, et al: Potential of the angiotensin receptor blockers (ARBs) telmisartan, irbesartan and candesartan for inhibiting the HMGB1/RAGE axis in prevention and acute treatment of stroke. Int J Mol Sci 14: 18899-18924, 2013.

37. Fu M, Zhou J, Xu J, et al: Olmesartan attenuates cardiac hypertrophy and improves cardiac diastolic function in spontaneously hypertensive rats through inhibition of calcineurin pathway. J Cardiovasc Pharmacol 63: 218-226, 2014.

38. Opie LH: Reperfusion injury and its pharmacologic modification. Circulation 80: 1049-1062, 1989.

39. Zhang X,WheelerD, Tang Y,etal:Calcium/calmodulin-dependent protein kinase (CaMK) IV mediates nucleocytoplasmic shuttling and release of HMGB1 during lipopolysaccharide stimulation of macrophages. J Immunol 181: 5015-5023, 2008.

40. Tang D, Kang R, Zeh HJ III and Lotze MT: High-mobility group box 1, oxidative stress and disease. Antioxid Redox Signal 14: 1315-1335, 2011.

41. Dai W,Hale SL,Kay GL,JyralaAJ and KlonerRA: Cardioprotective effects of angiotensin II type 1 receptor blockade with olmesartan on reperfusion injury in a rat myocardial ischemia-reperfusion injury model. Cardiovasc Ther 28: 30-37, 2010. 